\title{
Discovery of the Double Exponential Transformation and Its Developments
}

By

\author{
Masatake MorI*
}

\begin{abstract}
This article is mainly concerned how the double exponential formula for numerical integration was discovered and how it has been developed thereafter. For evaluation of $I=\int_{-1}^{1} f(x) d x$ H. Takahasi and M. Mori took advantage of the optimality of the trapezoidal formula over $(-\infty, \infty)$ with an equal mesh size and proposed in $1974 x=\phi(t)=\tanh \left(\frac{\pi}{2} \sinh t\right)$ as an optimal choice of variable transformation which transforms the original integral to $I=\int_{-\infty}^{\infty} f(\phi(t)) \phi^{\prime}(t) d t$. If the trapezoidal formula is applied to the transformed integral and the resulting infinite summation is properly truncated a quadrature formula $I \approx h \sum_{k=-n}^{n} f(\phi(k h)) \phi^{\prime}(k h)$ is obtained. Its error is expressed as $O(\exp (-C N / \log N))$ as a function of the number $N(=2 n+1)$ of function evaluations. Since the integrand decays double exponentially after the transformation it is called the double exponential (DE) formula. It is also shown that the formula is optimal in the sense that there is no quadrature formula obtained by variable transformation whose error decays faster than $O(\exp (-C N / \log N))$ as $N$ becomes large. Since the paper by Takahasi and Mori was published the DE formula has gradually come to be used widely in various fields of science and engineering. In fact we can find papers in which the DE formula is successfully used in the fields of molecular physics, fluid dynamics, statistics, civil engineering, financial engineering, in particular in the field of the boundary element method, and so on. The DE transformation has turned out to be also useful for evaluation of indefinite integrals, for solution of integral equations and for solution of ordinary differential equations, so that the scope of its applications is expected to spread also in the future.
\end{abstract}

Communicated by H. Okamoto. Received November 25, 2004.

2000 Mathematics Subject Classification(s): 01-08, 41A55, 65-03, 65R10, 65R20

*Department of Mathematical Sciences, Tokyo Denki University, Hatoyama, Hiki-gun, Saitama, 350-0394 Japan. 


\section{$\S 1$. Introduction}

History of numerical integration is long. In fact people have been using Simpson's formula since the time of T. Simpson (1710-1761) and Gauss' formula since the time of C. F. Gauss (1777-1855). However, these formulas can normally be used for integrands that are regular at the end points of integration. Although Gauss-Jacobi's formula has been known the type of singularity at the end points is quite limited. However, after computer appeared people tried to evaluate integrals with end-point singularity of an arbitrary power by computer.

This article is concerned with a discovery of a new formula for numerical integration which was a successful result of such a trial as well as its subsequent developments thereafter up to the symposium "Thirty Years of the Double Exponential Transforms" which was held on September 1st - 3rd 2004 at Research Institute for Mathematical Sciences, Kyoto University. The story of the discovery starts on the day on which the author visited the computer centre in University of Tokyo.

In 1969 Masatake Mori was working as a research associate for Faculty of Engineering, University of Tokyo. His research background was physics and, at that time, he belonged to Department of Applied Physics. He was working on atomic collision theory and his main daily task was to evaluate molecular integrals by computer.

One day in August 1969 Mori visited the computer centre in the university in order to see Toshiyasu Kunii ${ }^{1}$. Kunii was Mori's friend and working for the computer centre as a research associate. At this opportunity Kunii introduced Mori to Hidetosi Takahasi, the director of the computer centre.

Takahasi was very famous as a physicist in Japan. In fact, he used to be the president of Physical Soceity of Japan and was said to be "the physicist among physicists". At the same time he was also famous as a computer scientist in the dawn of computer science in Japan and was the president of Information Processing Society of Japan when Mori met him in 1969.

At this first interview Takahasi told Mori many things around computer science. Everything seemed to Mori quite novel and exciting. Among other things Takahasi persuaded Mori to work on error analysis in numerical integration of analytic function. It attracted Mori very much because numerical integration was his daily task and also analytic function theory was one of the mathematical tools which he knew how to handle. Actually in the faculty of engineering he was teaching complex function theory under the supervision of Hiroshi Fujita.

\footnotetext{
${ }^{1}$ The author begs every person appearing in the present paper a pardon to omit the title.
} 
On that day Takahasi and Mori talked for about one hour, and their cooperation thus started.

\section{$\S 2 . \quad$ Error Estimation in Numerical Integration}

\section{§2.1. Characteristic function of the error}

The idea of error analysis in numerical integration which Takahasi told Mori was as follows [74, 75, 76]. Since it plays a key role in the discovery of the double exponential formula we give here some details.

Suppose that we want to evaluate an integral

$$
I=\int_{a}^{b} f(x) d x
$$

where for simplicity we assume that $f(x)$ is analytic on $[a, b]$. Then, corresponding to this integral we consider a contour integral

$$
\frac{1}{2 \pi i} \oint_{C} \Psi(z) f(z) d z
$$

in the $z$ plane where $\Psi(z)$ is a logarithmic function defined by

$$
\Psi(z)=\log \frac{z-a}{z-b} .
$$

The path $C$ of integration is a contour surrounding the line segment $[a, b]$ in the positive direction in such a way that there is no singularity of $f(z)$ inside $C$ as shown in Fig.1. If we deform the path $C$ infinitely close to $[a, b]$ we immediately have

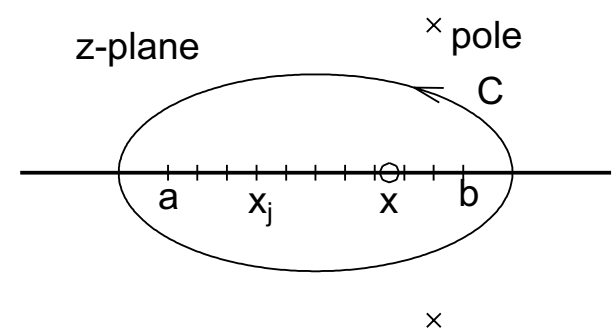

Figure 1. The path $C$

$$
\frac{1}{2 \pi i} \oint_{C} \Psi(z) f(z) d z=\int_{a}^{b} f(x) d x .
$$


On the other hand suppose that we use a quadrature formula with points $x_{k}$ and weights $A_{k}$ in order to evaluate approximately the integral (2.1):

$$
I_{n}=\sum_{k=1}^{n} A_{k} f\left(x_{k}\right) .
$$

Then, corresponding to this quadrature formula we consider a contour integral

$$
\frac{1}{2 \pi i} \oint_{C} \Psi_{n}(z) f(z) d z
$$

where $\Psi_{n}(z)$ is a rational function defined by

$$
\Psi_{n}(z)=\sum_{k=1}^{n} \frac{A_{k}}{z-x_{k}}
$$

and the path $C$ is the same as is given in (2.2). Since we assumed that $f(z)$ does not have any singularity inside $C$ the only singular points of $\Psi_{n}(z) f(z)$ inside $C$ are the simple poles $x_{k}, k=1,2, \ldots, n$ of $\Psi_{n}(z)$, and hence by the residue theorem we immediately have

$$
\frac{1}{2 \pi i} \oint_{C} \Psi_{n}(z) f(z) d z=\sum_{k=1}^{n} A_{k} f\left(x_{k}\right) .
$$

Therefore we see that the error of the quadrature formula (2.5) is expressed in terms of a contour integral

$$
\Delta I_{n}=I-I_{n}=\frac{1}{2 \pi i} \oint_{C} \Phi_{n}(z) f(z) d z
$$

where $\Phi_{n}(z)$ is defined by

$$
\Phi_{n}(z)=\Psi(z)-\Psi_{n}(z)=\log \frac{z-a}{z-b}-\sum_{k=1}^{n} \frac{A_{k}}{z-x_{k}}
$$

and the contour $C$ is given as above. We call $\Phi_{n}(z)$ the characteristic function of the error because it characterizes the error of the formula independently of the integrand $f(x)$.

Takahasi told Mori that it would be helpful to plot a contour map of $\left|\Phi_{n}(z)\right|$ for the purpose of actual estimation of the error. Since Takahasi was the director of the computer centre Mori was able to take full advantage of accessibility to the computer system including an XY plotter. A few days later Mori completed a program and obtained successfully contour maps of $\left|\Phi_{n}(z)\right|$ for several typical quadrature formulas [22]. 
In Fig.2 the contour map of $\left|\Phi_{n}(z)\right|$ for Simpson's formula over $[-1,1]$ with a mesh size $h=0.1(n=21)$ is shown. As seen from this figure the absolute value $\left|\Phi_{n}(z)\right|$ decreases quickly as $z$ goes far from the interval $[a, b]$ of integration in the $z$ plane. This behavior is generally observed in good quadrature formulas. In fact, points and weights of formulas we usually use are chosen in such a way that $\left|\Phi_{n}(z)\right|$ becomes as small as possible for large $|z|$. In other words the approximation problem of integration by a quadrature formula can be replaced by a problem of approximation of the logarithmic function $\log (z-a) /(z-b)$ by a rational function $\sum_{k=1}^{n} A_{k} /\left(z-x_{k}\right)$ at large $|z|$ as seen from (2.10).

This figure can be used for practical estimation of the error. For easy understanding we show here a very simple example. Suppose that we want to

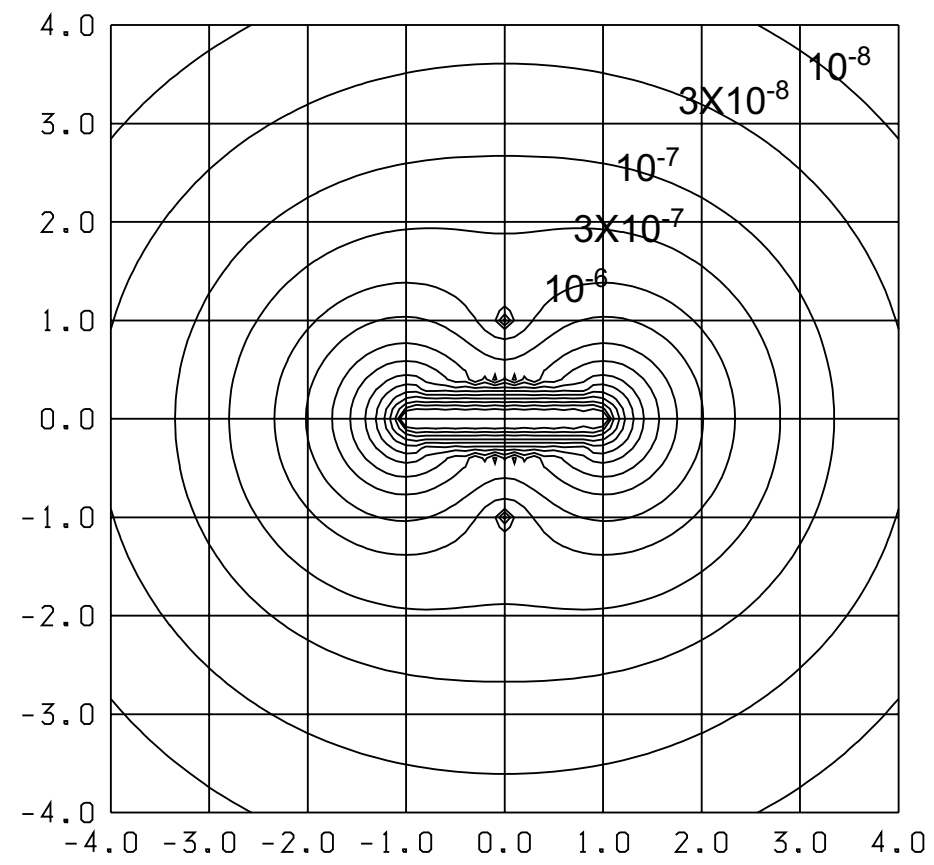

Figure 2. $\left|\Phi_{n}(z)\right|$ for Simpson's formula $(h=0.1)$

evaluate

$$
I=\int_{-1}^{1} \frac{1}{x-2} d x=-\log 3=-1.098612288 \cdots
$$


using Simpson's formula with $h=0.1$. Then, since $\operatorname{Res}\left(\frac{1}{x-2}, 2\right)=1$ and $\left|\Phi_{n}(2)\right| \approx 3 \times 10^{-6}$ from Fig.2, we have an estimate of the error from (2.9) and by the residue theorem

$$
\left|\Delta I_{n}\right|=\left|\Phi_{n}(2) \operatorname{Res}\left(\frac{1}{x-2}, 2\right)\right| \approx 3 \times 10^{-6} .
$$

If we actually compute (2.11) using Simpson's formula we will get a result $I_{n}=-1.098615504 \cdots$ whose error is about $-3 \times 10^{-6}$. When the integrand is not a rational function we may use the saddle point method as an alternative method [23].

\section{§2.2. The trapezoidal formula over $(-\infty, \infty)$}

Suppose that we want to evaluate an integral

$$
I=\int_{-\infty}^{\infty} g(t) d t
$$

where $g(t)$ is an analytic function over $(-\infty, \infty)$. Then, it has been known among numerical analysts that if we apply the trapezoidal formula

$$
I_{h}=h \sum_{k=-\infty}^{\infty} g(k h)
$$

with an equal mesh size to this integral we will usually get a result with very high accuracy $[4,58,60]$. The very high accuracy, which might seem to be against common knowledge about the trapezoidal formula, stems from an optimality of the trapezoidal formula applied to integrals of analytic function over $(-\infty, \infty)$.

Takahasi and Mori gave a theorem on the optimality from the stand point of the characteristic function of the error which will be outlined below [34, 75]. First we note that the error of the formula (2.14) can be expressed in terms of a contour integral

$$
I-I_{h}=\Delta I_{h}=\frac{1}{2 \pi i} \int_{\hat{C}} \hat{\Phi}_{h}(w) g(w) d w
$$

where $\hat{\Phi}_{h}(w)$ is a function defined by

$$
\hat{\Phi}_{h}(w)=\left\{\begin{array}{l}
\frac{-2 \pi i}{1-\exp \left(-\frac{2 \pi i}{h} w\right)} ; \operatorname{Im} w>0 \\
\frac{+2 \pi i}{1-\exp \left(+\frac{2 \pi i}{h} w\right)} ; \operatorname{Im} w<0
\end{array}\right.
$$


and the path $\hat{C}$ of integration consists of two infinite curves, one of which runs to the left in the upper half plane and the other runs to the right in the lower half plane in such a way that there is no singularities of $g(w)$ between these two curves. The function $\hat{\Phi}_{h}(w)$ is nothing but the characteristic function of the error of the trapezoidal formula (2.14). It is not difficult to prove (2.15) if we note that

$$
\hat{\Phi}_{h}(w)= \begin{cases}-\pi i-\pi \cot \frac{\pi}{h} w ; & \operatorname{Im} w>0 \\ +\pi i-\pi \cot \frac{\pi}{h} w ; & \operatorname{Im} w<0\end{cases}
$$

as well as the partial fraction expansion of the cot function

$$
\pi \cot \frac{\pi}{h} w=h\left\{\frac{1}{w}+\sum_{k=1}^{\infty}\left(\frac{1}{w-k h}+\frac{1}{w+k h}\right)\right\} .
$$

If $w$ is far from the real axis the function (2.16) behaves

$$
\left|\hat{\Phi}_{h}(w)\right| \approx 2 \pi \exp \left(-\frac{2 \pi}{h}|\operatorname{Im} w|\right) .
$$

This means that if the mesh size $h$ is small enough $\left|\hat{\Phi}_{h}(w)\right|$ decays exponentially with an exponent $2 \pi / h$ as $|\operatorname{Im} w|$ becomes large, and hence the contour map of the characteristic function $\left|\hat{\Phi}_{h}(w)\right|$ consists of lines parallel to the real axis as long as the contour is not too close to the real axis. In Fig. 3 the contour map of $\left|\hat{\Phi}_{h}(w)\right| /(2 \pi)$ defined by (2.16) with $h=0.1$ is shown.

\section{$\S 2.3$. Optimality of the trapezoidal formula}

Now we generalize the idea of the characteristic function for the trapezoidal formula described above to other formulas for numerical integration over $(-\infty, \infty)$. Consider a formula

$$
I_{\mathrm{A}}=\sum_{k=-\infty}^{\infty} A_{k} g\left(x_{k}\right)
$$

where $x_{k}$ is the $k$ th point and $A_{k}$ is the corresponding weight. The subscript A indicates that $I_{\mathrm{A}}$ is an approximation to $I$. Here we assume that the error can be written

$$
\Delta I_{\mathrm{A}}=I-I_{\mathrm{A}}=\frac{1}{2 \pi i} \int_{\hat{C}} \hat{\Phi}_{\mathrm{A}}(w) g(w) d w
$$




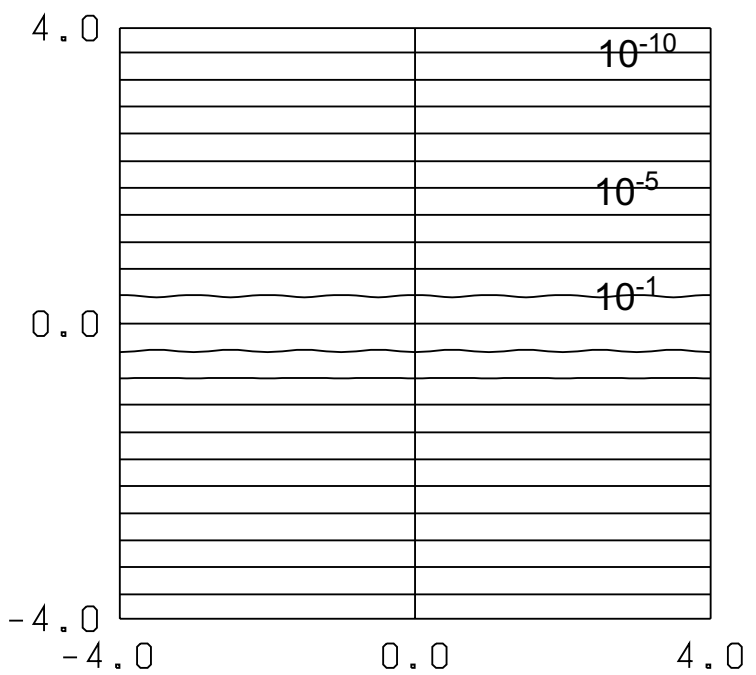

Figure 3. $\left|\hat{\Phi}_{h}(z)\right| /(2 \pi)$ for trapezoidal formula $(h=0.1)$

like (2.15). In fact, typical quadrature formulas for integrals over $(-\infty, \infty)$ including the trapezoidal formula, can be expressed in this form. $\hat{\Phi}_{\mathrm{A}}(w)$ is the characteristic function of the error for the formula (2.20). First we note that $-\partial / \partial v \log \left|\hat{\Phi}_{\mathrm{A}}(w)\right|, v=|\operatorname{Im} w|$ is the exponent of the decay of $\left|\hat{\Phi}_{\mathrm{A}}(w)\right|$ as a function of the distance $v$ from the real axis. In case of the trapezoidal formula $-\partial / \partial v \log \left|\hat{\Phi}_{\mathrm{A}}(w)\right| \approx 2 \pi / h$ holds. Then we define the average exponent of the characteristic function along the straight line parallel to the real axis whose distance from the real axis is $\ell$ :

$$
r(\ell)=\lim _{R \rightarrow \infty} \frac{1}{2 R} \int_{-R+i \ell}^{R+i \ell}\left\{-\frac{\partial}{\partial v} \log \left|\hat{\Phi}_{\mathrm{A}}(w)\right|\right\} d w, \quad v=\operatorname{Im} w .
$$

Furthermore we define the asymptotic average exponent by

$$
r=\lim _{|\ell| \rightarrow \infty} r(\ell) .
$$

Then the main theorem can be stated as follows. Under the assumptions given above, among formulas $I_{A}$ whose average number of the points $\left\{x_{k}\right\}$ per unit length is constant $\nu_{\mathrm{P}}$, the trapezoidal formula $I_{h}$ with the mesh size $h=1 / \nu_{\mathrm{P}}$ is 
optimal in the sense that the asymptotic average exponent $r$ attains its possible maximum

$$
r_{\max }=2 \pi \nu_{\mathrm{P}}=\frac{2 \pi}{h} .
$$

In order to prove this optimality Takahasi and Mori modified the path of integration of (2.22) and applied the principle of argument to obtain

$$
r=\lim _{\ell \rightarrow \infty} \lim _{R \rightarrow \infty} \frac{1}{2 R} \int_{-R+i \ell}^{R+i \ell}\left\{-\frac{\partial}{\partial v} \log \left|\hat{\Phi}_{\mathrm{A}}(w)\right|\right\} d w=2 \pi\left(\nu_{\mathrm{P}}-\nu_{\mathrm{Z}}\right),
$$

where $\nu_{\mathrm{Z}}$ denotes the average number of zeros of $\hat{\Phi}_{\mathrm{A}}(w)$ per unit length to the direction parallel to the real axis, while $\nu_{\mathrm{P}}$ is the average number of poles since $x_{k}$ corresponds to a pole of $\hat{\Phi}_{\mathrm{A}}(w)$. From $(2.25)$ we have

$$
r=2 \pi\left(\nu_{\mathrm{P}}-\nu_{\mathrm{Z}}\right) \leq 2 \pi \nu_{\mathrm{P}}=\text { constant }
$$

which means that, if there is a formula for which $\nu_{\mathrm{Z}}=0$, it is optimal. On the other hand, the characteristic function $\hat{\Phi}_{h}(w)$ of the trapezoidal formula does not have zeros in the finite $w$ plane, so that $\nu_{\mathrm{Z}}=0$ holds. Therefore we conclude that the trapezoidal formula is optimal. See [75] or [23] for details of the proof.

As seen above the asymptotic average exponent for the trapezoidal formula with mesh size $h$ is $r=2 \pi / h$ and it attains the optimal value. In contrast, in case of Simpson's formula the asymptotic average exponent is $\pi / h$ which is just the half of that of the trapezoidal formula with the same mesh size.

Mori took advantage of this optimality and proposed an efficient method for high precision evaluation of the error function [26]. See also [17] for a preceding work based on a similar idea. Incidentally he proved the optimality of the trapezoidal formula for integrals of a periodic analytic function in a similar way as shown above [21].

The optimality of the trapezoidal formula for integrals of an analytic function over $(-\infty, \infty)$ proved as above will turn out to play a fundamental role in the process of the discovery of the double exponential formula.

It should be noted that the optimality of the trapezoidal formula has been discussed by a number of researchers. In particular there have been a trend among mathematicians in Europe, US and Canada to discuss about the optimality via the Hardy space. See [13] and references therein.

In about three months since Mori first met Takahasi they completed their joint research on the error estimation in numerical integration described above. 
They reported the results in a symposium organized by Research Institute for Mathematical Sciences, Kyoto University [74]. Hereafter we abbreviate the institute to RIMS and also call the symposium RIMS symposium. This RIMS symposium was held from 5 th until 7 th November 1969 . Takahasi spoke about the basic idea of the error estimation and Mori gave a detailed analysis and results of numerical experiments. Their talk seemed to have attracted many people's interest.

Soon after the symposium Mori was offered a position of associate professor at RIMS. He accepted the offer and moved to the institute in March 1970. At RIMS he could exclusively devote himself to the research of numerical analysis and, although he and Takahasi were separated at Kyoto and at Tokyo, there was no obstruction in their joint research activities.

They submitted a paper on the error analysis stated above to "Report of Computer Centre, University of Tokyo" [75]. It was a new journal published by the computer centre and Takahasi thought as the director that it was his duty to encourage submission of good papers to the journal. Although their paper was published in 1970, circulation of the new journal was not good and soon later Mori was blamed by several researchers that he should not have submitted their work to such an obscure journal. In order to recover from this situation Mori delivered copies of the paper to several representative numerical analysts. Philip Rabinowitz was one of them and he spared a few lines for their error analysis in "Methods of Numerical Integration" which he and Philip J. Davis jointly published in 1975 [4]. A shorter version of [75] was published in [76].

After Mori moved to RIMS he found that their idea of the characteristic function is closely related to the theory of hyperfunction by Mikio Sato, a professor of RIMS. In fact, a linear functional over analytic functions is defined as a hyperfunction, and the error of numerical integration is a linear functional. The characteristic function of the error is nothing but a defining function of the corresponding hyperfunction, the error of numerical integration [57]. Mori has an opportunity to discuss about it with Takahiro Kawai, one of Sato's young co-researchers, and in accordance with Kawai's suggestion Mori gave a talk on the error analysis at a RIMS symposium on partial differential equations and hyperfunctions on March 22 in 1971 [19]. This was a rare and precious chance to publicize their research work on numerical integration among pure mathematicians inside Japan. 


\section{§3. Variable Transformation in Numerical Integration}

\section{§3.1. The IMT-rule}

At the RIMS symposium in November 1969 another interesting research result was reported. It was on a new quadrature formula based on a variable transformation by Masao Iri, Sigeiti Moriguti and Yoshimitsu Takasawa [8]. It is for an integral over $(0,1)$ of an analytic function $f(x)$ which may have end-point singularity:

$$
I=\int_{0}^{1} f(x) d x .
$$

They applied a variable transformation

$$
\begin{aligned}
x=\phi(t) & =\frac{1}{Q} \int_{0}^{t} \exp \left[-\left(\frac{1}{s}+\frac{1}{1-s}\right)\right] d s, \\
Q & =\int_{0}^{1} \exp \left[-\left(\frac{1}{s}+\frac{1}{1-s}\right)\right] d s,
\end{aligned}
$$

to the integral and used the trapezpodal formula by dividing the interval $(0,1)$ into $N$ subintervals with an equal mesh size $h=1 / N$ to obtain

$$
I_{N}=h \sum_{k=0}^{N} f(\phi(k h)) \phi^{\prime}(k h), \quad h=1 / N .
$$

The function $\phi(t)$ maps the original interval $(0,1)$ onto itself. The function values as well as all the derivatives in (3.4) vanish at both end points, and hence from Euler-Maclaurin's formula we can expect that the error produced by the formula is very small. Actually they showed that the error of the formula is expressed

$$
I-I_{N}=O(\exp (-C \sqrt{N}))
$$

as a function of $N$.

Incidentally, when Takahasi and Mori were preparing the manuscript of their paper on error estimation [75] they gave a brief introduction of this formula because they wanted to add a contour map of the characteristic function of the error for this new formula in their paper. Later in 1973 they described again about it in [78] and called it the IMT-rule after the initials of the authors. After the paper [78] was published Mori sent again a copy to Philip Rabinowitz. It seemed to be quite timely because Davis and Rabinowitz gave a 
detailed description about the IMT-rule in their book "Methods of Numerical Integration" [4]. Later in 1987 a special issue of Journal of Computational and Applied Mathematics devoted to numerical integration was published whose editors were Mori and Robert Piessens. The original paper in Japanese by Iri, Moriguti and Takasawa [8] was translated into English by the authors themselves and republished in the special issue [9].

\section{§3.2. Variable transformation onto $(-\infty, \infty)$ and application of the trapezoidal formula}

Let us return to the RIMS symposium in November 1969. Takahasi and Mori were greatly stimulated by the IMT-rule when it was reported in the symposium, and immediately after they went back to Tokyo they began to study about quadrature formulas using a variable transformation. As already pointed out Mori moved to RIMS in March 1970 and they were separated at Tokyo and at Kyoto. However, Mori could frequently visit University of Tokyo to see Takahasi and their joint work proceeded smoothly.

Since they had already proved the optimality of the trapezoidal formula over $(-\infty, \infty)$ it was quite natural that they started with a variable transformation which maps the original interval of integration onto $(-\infty, \infty)$ and applied the trapezoidal formula. In addition they thought that if they transform the original integral into another finite interval like the IMT-rule essential singular points appear in the finite complex plane and that the error analysis would be more complicated than in the case of the transformation onto $(-\infty, \infty)$ where essential singular points appear only at infinity.

Since we can generalize the discussion to the integral $\int_{a}^{b} f(\xi) d \xi$ by applying a transformation $\xi=(b-a) x / 2+(b+a) / 2$, we will confine ourselves to the case $a=-1, b=1$ without loss of generality throughout this paper. And hence we suppose that the given integral is over $(-1,1)$ :

$$
I=\int_{-1}^{1} f(x) d x .
$$

Then we transform the integral (3.6) using a function

$$
x=\phi(t)
$$

which we assume is analytic over $-\infty<t<\infty$ and satisfies

$$
\phi(-\infty)=-1, \phi(+\infty)=1
$$


to obtain

$$
I=\int_{-\infty}^{\infty} f(\phi(t)) \phi^{\prime}(t) d t
$$

In order to evaluate the integral (3.9) we employ the trapezoidal formula

$$
I_{h}=h \sum_{k=-\infty}^{\infty} f(\phi(k h)) \phi^{\prime}(k h)
$$

with an equal mesh size $h$ since we know its optimality. The integrand $f(z)$ in (3.6) has at least one singular point on the $z$ plane including $z=\infty$. This point usually is mapped by $z=\phi(w)$ onto an infinite array of points in the $w$ plane, i.e. $f(\phi(w)) \phi^{\prime}(w)$ after the transformation has an infinite array of singular points. Let $d$ be the minimum distance between these points and the real axis. In other words we assume that $f(\phi(w)) \phi^{\prime}(w)$ is regular in the strip region

$$
|\operatorname{Im} w|<d
$$

Then from (2.15) and (2.19) we have

$$
I=\int_{-\infty}^{\infty} f(\phi(t)) \phi^{\prime}(t) d t=h \sum_{k=-\infty}^{\infty} f(\phi(k h)) \phi^{\prime}(k h)+\Delta I_{h}
$$

where

$$
\Delta I_{h}=I-I_{h}=O\left(\exp \left(-\frac{2 \pi d}{h}\right)\right)
$$

We call this error $\Delta I_{h}$ the discretization error.

\section{§3.3. Truncation of infinite summation}

It is important to note that when we actually compute the infinite summation $I_{h}$ we must truncate it into a finite sum

$$
I_{h}=I_{h}^{(N)}+\varepsilon_{t},
$$

where

$$
I_{h}^{(N)}=h \sum_{k=-n}^{n} f(\phi(k h)) \phi^{\prime}(k h)
$$


and

$$
\varepsilon_{t}=h \sum_{k=-\infty}^{-n} f(\phi(k h)) \phi^{\prime}(k h)+h \sum_{k=n}^{\infty} f(\phi(k h)) \phi^{\prime}(k h) .
$$

Here we assume that we truncate the summation, for simplicity, at the same number of terms $n$ on both positive and negative sides of $k$, so that the total number of function evaluations is

$$
N=2 n+1
$$

We call the error $\varepsilon_{t}$ the truncation error.

For the truncation to be relevant we should truncate the infinite summation (3.10) in such a way that the discretization error $\Delta I_{h}$ is equal to the truncation error $\varepsilon_{t}$, i.e.

$$
\Delta I_{h}=\varepsilon_{t}
$$

Thus we hereafter denote the overall error as $\Delta I_{h}^{(N)}$ and write

$$
I=I_{h}^{(N)}+\Delta I_{h}^{(N)}
$$

where $\Delta I_{h}^{(N)}=\Delta I_{h}+\varepsilon_{t}=2 \Delta I_{h}=2 \varepsilon_{t}$ as long as we carry out truncation according to (3.18).

\section{§3.4. Choice of transformation}

Since there still remains a choice of $\phi(t)$ Takahasi and Mori examined several types of functions for $\phi(t)$ which maps $(-1,1)$ onto $(-\infty, \infty)$. Among these functions we select here two functions, $\tanh t$ and erf $t$. Although they assumed that the original integrand $f(x)$ is expressed $f(x)=\left(1-x^{2}\right)^{-\alpha} f_{1}(x), \alpha<1$ we will follow their analysis by taking $\alpha=0$ for simplicity here.

First consider

$$
x=\tanh t
$$

Since $d=\pi / 2$ for $d$ in (3.11) in this case because $\phi^{\prime}(w)=1 / \cosh w$ has singular points $w= \pm \pi i / 2$, we see that $\Delta I_{h}=O\left(\exp \left(-\pi^{2} / h\right)\right)$. Therefore from $\varepsilon_{t}=O(\exp (-N h))$ and from $(3.18)$ we have a relation between $h$ and $N$

$$
h=\frac{\pi}{\sqrt{N}} .
$$


Substituting (3.21) into (3.19) we have an error expression for $\Delta I_{h}^{(N)}$ in terms of the number $N$ of function evaluations, and finally we obtain

$$
I=h \sum_{k=-n}^{n} f(\tanh k h) \frac{1}{\cosh ^{2} k h}+O(\exp (-\pi \sqrt{N})) .
$$

Next consider

$$
x=\operatorname{erf} t=\frac{2}{\sqrt{\pi}} \int_{0}^{t} \exp \left(-\tau^{2}\right) d \tau .
$$

In a similar manner as in the case of $\tanh t$ we have

$$
I=\frac{2}{\sqrt{\pi}} h \sum_{k=-n}^{n} f(\operatorname{erf} k h) \exp \left(-k^{2} h^{2}\right)+O\left(\exp \left(-3.4 N^{2 / 3}\right)\right) .
$$

If we compare (3.22) and (3.24) we see that the latter formula based on $x=\operatorname{erf} t$ is superior to the former formula based on $x=\tanh t$ because the error of the latter formula decreases more quickly than that of the former one as $N$ becomes large. Note that the decay of $\phi^{\prime}(t)=\exp \left(-t^{2}\right)$ is faster than that of $\phi^{\prime}(t)=1 / \cosh t \approx 2 \exp (-|t|)$. This seems to indicate that if the decay of $\phi^{\prime}(t)$ becomes faster the efficiency of the resulting formula increases.

Takahasi and Mori reported orally the result of analysis described above at a RIMS symposium in November 1971 [77], and submitted a paper to Numerische Mathematik. In the course of refereeing process the referee brought the paper by C. Schwartz [58] into their notice which was a significant report of a similar work prior to their analysis. Their paper was published in 1973 [78].

\section{§4. Discovery of the Double Exponential Transformation}

\section{§4.1. Aiming at the optimal transformation}

For Takahasi and Mori their result reported in 1973 [78] was an interim one because they believed that there would be an optimal transformation which results in a formula that gives the highest accuracy with the minimum number of function evaluations. They continued their research and pursued the optimal transformation in the following way.

Suppose that we choose a transformation $x=\phi(t)$ which makes the decay of the integrand $f(\phi(t)) \phi^{\prime}(t)$ fast as $|t| \rightarrow \infty$ and that we keep $h$ at a moderate value. Then the truncation error $\varepsilon_{t}$ given by (3.16) becomes small when we truncate the summation

$$
I_{h}=h \sum_{k=-\infty}^{\infty} f(\phi(k h)) \phi^{\prime}(k h)
$$


at some moderate value of $k= \pm n$. However, if the transformation $x=\phi(t)$ is such that it makes the decay of the integrand too fast, the mesh size $h$ becomes relatively large compared with the variation of the integrand $f(\phi(t)) \phi^{\prime}(t)$, so that the discretization error $\Delta I_{h}$ in (3.13) will become large.

On the contrary, if we choose a transformation which makes the decay of the integrand $f(\phi(t)) \phi^{\prime}(t)$ slow, then the mesh size $h$ becomes relatively small compared with the variation of $f(\phi(t)) \phi^{\prime}(t)$, so that the discretization error $\Delta I_{h}$ becomes small. However, if we truncate the summation at the same $k= \pm n$ as in the case of fast decay, the term is not yet small enough and the truncation error $\varepsilon_{t}$ becomes large.

From this observation we see that the error becomes large in both cases, i.e. in the case where the decay of the integrand $f(\phi(t)) \phi^{\prime}(t)$ is too fast and in the case where it is too slow. Consequently, there should be an optimal decay between these two cases.

\section{$\S 4.2$ Discovery of the double exponential transformation}

Now we start again from an integral of the form

$$
I=\int_{-1}^{1} f(x) d x
$$

and search for the transformation $x=\phi(t)$ that makes the error converge fastest to zero as $N=2 n+1$, the number of function evaluations, becomes large under the condition (3.18). For that purpose we choose a sequence of transformations from the one that gives a slow decay to the one that gives a faster decay, and see how the error changes.

First we choose a transformation

$$
x=\phi(t)=\tanh t^{\rho}, \quad \phi^{\prime}(t)=\frac{\rho t^{\rho-1}}{\cosh ^{2} t^{\rho}}, \quad \rho=1,3,5, \cdots .
$$

For each $\rho$ there corresponds to a formula, and as $\rho$ increases the derivative

$$
\phi^{\prime}(t)=\frac{\rho t^{\rho-1}}{\cosh ^{2} t^{\rho}} \approx O\left(\exp \left(-t^{\rho}\right)\right) \quad \text { as } \quad|t| \rightarrow \infty
$$

decays faster at large $|t|$. Note that this decay is single exponential and $\rho=1$ corresponds to (3.20). If we follow the criterion (3.18) for truncation we have a relation

$$
h=\left(2^{\rho} \pi d\right)^{\frac{1}{\rho+1}} N^{-\frac{\rho}{\rho+1}}
$$


between $h$ and $N$ and, substituting it into (3.12) and (3.13), we obtain for each $\rho$ a formula

$$
I_{h}^{(N)}=h \sum_{k=-n}^{n} f\left(\tanh (k h)^{\rho}\right) \frac{\rho k^{\rho-1} h^{\rho-1}}{\cosh ^{2}\left((k h)^{\rho}\right)}
$$

and an error representation

$$
\left|\Delta I_{h}^{(N)}\right|=O\left(\exp \left(-\left(2^{\frac{1}{\rho}} \pi d N\right)^{\frac{\rho}{\rho+1}} N^{\frac{\rho}{\rho+1}}\right)\right)
$$

where $I=I_{h}^{(N)}+\Delta I_{h}^{(N)}$. We see from (4.7) that as $\rho$ increases the error $\left|\Delta I_{h}^{(N)}\right|$ decreases as is expected from the discussion at the end of the previous section.

A faster decay next to single exponential is double exponential, so that in order to see how the error behaves, we choose here a transformation which results in a double exponential decay of the integrand:

$$
x=\phi(t)=\tanh \left(\frac{\pi}{2} \sinh t\right) .
$$

In this choice of function

$$
\phi^{\prime}(t)=\frac{\frac{\pi}{2} \cosh t}{\cosh ^{2}\left(\frac{\pi}{2} \sinh t\right)} \approx O\left(\exp \left(-\frac{\pi}{2} \exp |t|\right)\right) \text { as } \quad|t| \rightarrow \infty
$$

holds. Due to this double exponential decay we call (4.8) the double exponential transformation, abbreviated as the DE transformation. If we truncate (4.1) at $k= \pm n$ in such a way that (3.18) is satisfied, we see from (4.9) that $\varepsilon_{t} \approx$ $\exp \left(-\frac{\pi}{2} \exp (n h)\right)$ holds and have

$$
h=\frac{2}{N} \log (2 d N)
$$

from (3.18) where $N=2 n+1$. This gives a formula

$$
I_{h}^{(N)}=h \sum_{k=-n}^{n} f\left(\tanh \left(\frac{\pi}{2} \sinh k h\right)\right) \frac{\frac{\pi}{2} \cosh k h}{\cosh ^{2}\left(\frac{\pi}{2} \sinh k h\right)}
$$

and its error representation

$$
\left|\Delta I_{h}^{(N)}\right|=O\left(\exp \left(-\frac{\pi d N}{\log (2 d N)}\right)\right)
$$

where $I=I_{h}^{(N)}+\Delta I_{h}^{(N)}$. We call (4.11) the double exponential formula, abbreviated as the DE formula, after the decay (4.9). We see that as $N$ increases this error becomes small much faster than (4.7). 
Now we choose a transformation

$$
x=\phi(t)=\tanh \left(\frac{\pi}{2} \sinh t^{3}\right)
$$

which results in a faster decay of $\phi^{\prime}(t)$ for large $|t|$ than double exponential given by (4.9). In this case we see from (2.15) that the discretization error becomes

$$
\Delta I_{h}=\frac{1}{2 \pi i} \int_{\hat{C}} \hat{\Phi}_{h}(w) f\left(\tanh \left(\frac{\pi}{2} \sinh w^{3}\right)\right) \frac{\frac{3 \pi}{2} w^{2} \cosh w^{3}}{\cosh ^{2}\left(\frac{\pi}{2} \sinh w^{3}\right)} d w .
$$

However, the singular points of the integrand in (4.14), i.e. the zeros of the denominator $\cosh ^{2}\left(\frac{\pi}{2} \sinh w^{3}\right)$, approach arbitrarily close to the real axis as $|\operatorname{Re} w|$ becomes large, and hence the error become larger than (3.13).

Incidentally, before they carried out comparison among transformations based on the number $N$ of function evaluations described above, Takahasi had given in advance a conclusion by his sharp intuition. Suppose that several factors have some contribution to an object. Then, the object attains its optimal state when every factor contributes equally to the object. Based on this general idea of optimality Takahasi predicted that the transformation (4.8) will give an optimal formula because the derivative of (4.8) has an infinite array of singular points which lie parallel to the real axis and that their contributions to the error are all equal.

From the analysis described above Takahasi and Mori concluded the optimality of the DE transformation. In order to confirm the optimality of the DE transformation numerically they chose the integral

$$
I=\int_{-1}^{1} \frac{d x}{(x-2)(1-x)^{1 / 4}(1+x)^{3 / 4}}(=-1.9490 \cdots)
$$

as an example and evaluated them using the following four transformations:

$$
\begin{aligned}
& \text { a. } x=\phi(t)=\tanh t, \quad \phi^{\prime}(t)=\frac{1}{\cosh ^{2} t} \\
& \text { b. } x=\phi(t)=\operatorname{erf} t=\frac{2}{\sqrt{\pi}} \int_{0}^{t} \exp \left(-\tau^{2}\right) d \tau, \quad \phi^{\prime}(t)=\frac{2}{\sqrt{\pi}} \exp \left(-t^{2}\right) \\
& \text { c. } x=\phi(t)=\tanh \left(\frac{\pi}{2} \sinh t\right), \quad \phi^{\prime}(t)=\frac{\frac{\pi}{2} \cosh t}{\cosh ^{2}\left(\frac{\pi}{2} \sinh t\right)} \\
& \text { d. } x=\phi(t)=\tanh \left(\frac{\pi}{2} \sinh t^{3}\right), \quad \phi^{\prime}(t)=\frac{\frac{3 \pi}{2} t^{2} \cosh t^{3}}{\cosh ^{2}\left(\frac{\pi}{2} \sinh t^{3}\right)} .
\end{aligned}
$$

The result is shown in Fig.4. The abscissa is the number $N$ of function eval- 


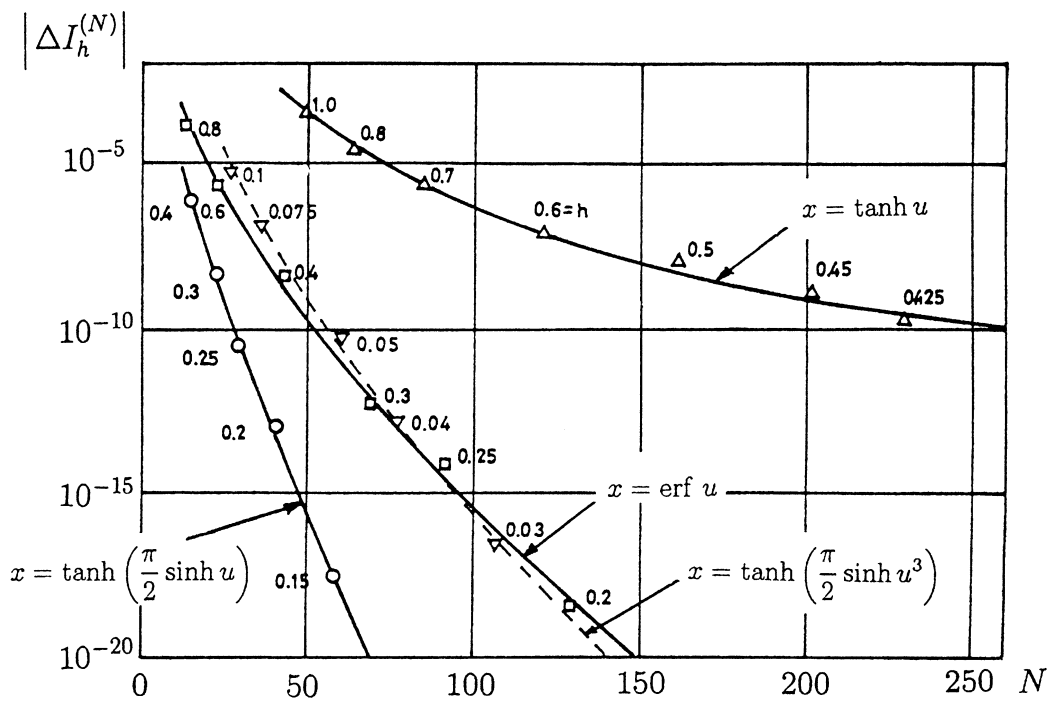

Figure 4. Comparison of the efficiency of several variable transformations for the integral $\int_{-1}^{1} d x /\left\{(x-2)(1-x)^{1 / 4}(1+x)^{3 / 4}\right\}$

uations and the ordinate is the absolute error $\left|I-I_{h}^{(N)}\right|$ in logarithmic scale actually computed. The number attached to each curve in the figure is the mesh size $h$ used for actual computation. Transformation c gives the DE formula. From this figure we see that the efficiency becomes higher as the decay of $\left|\phi^{\prime}(t)\right|$ is faster, and it attains the highest when the DE transformation is applied. Then, as the decay becomes faster than double exponential the efficiency turns to be lower.

Thus, Takahasi and Mori were convinced of the optimality of the DE transformation and presented the result orally at a RIMS symposium in 1973 [79] and published as a paper in Publ. RIMS in 1974 [80].

\section{§4.3. Application of the DE transformation to other types of integrals}

The idea of the DE transformation can be applied to various kinds of integrals. Takahasi and Mori gave some examples other than (4.8) in their paper in 1974 [80]. We list here typical types of integrals and corresponding 
relevant transformations:

$$
\begin{array}{rlrl}
\text { a. } & I=\int_{0}^{\infty} f_{1}(x) d x, \quad x=\exp \left(\frac{\pi}{2} \sinh t\right) \\
\text { b. } \quad I=\int_{0}^{\infty} f_{2}(x) e^{-x} d x, \quad x=\exp (t-\exp (-t)) \\
\text { c. } \quad I=\int_{-\infty}^{\infty} f_{3}(x) d x, \quad x=\sinh \left(\frac{\pi}{2} \sinh t\right) .
\end{array}
$$

$f_{1}(x)$ is an algebraic function decaying slowly at large positive $x, f_{2}(x)$ is an algebraic function decaying slowly at large positive $x$, and $f_{3}(x)$ is an algebraic function decaying slowly both at large $\pm x$. In b the integrand $f_{2}(x) e^{-x}$ decays exponentially at large positive $x$ as it is, so that what we need is to add one more exponential decay at large positive $x$.

Mori also proposed a formula based on an asymmetric transformation

$$
x=\phi(t)=\tanh (A \exp (a t)-B \exp (-b t))
$$

to meet an asymmetric distribution of singularities in the integrand [30].

\section{§4.4. Comments on the IMT-rule}

After the DE formula was established Mori proposed in 1977 an IMT-type DE transformation $[24,25]$ which maps $(-1,1)$ onto itself. He found that the error behavior of the resulting formula is $\exp \left(-C N /(\log N)^{2}\right)$ which indicates that this formula does not beat the efficiency of the proper DE formula. Although the number $N$ of function evaluations is essentially finite the trapezoidal sum must be truncated at some smaller value of $k$ in actual computation. Also Murota and Iri tried to improve the efficiency of the IMT-rule by tuning parameters and by repeated application of the IMT-transformation [40, 41]. However, although they could bring the efficiency very close to that of the DE formula, they were not successful in finding a formula whose efficiency goes beyond that of the DE formula.

\section{§4.5. Remarks on implimentation}

In order to use the DE transformation efficiently we usually need special attention in coding. One of the pitfalls which users often fall into is overflow due to the term $\cosh ^{2}\left(\frac{\pi}{2} \sinh t\right)$ in the denominator of (4.9) as is mentioned by Mori [27] and also by U.V. Ahie et al. in their report on a comparison of quadrature formulas based on variable transformation for double and triple 
singular integrals [1]. Note that $\cosh ((\pi / 2) \sinh 4.74)=5.35 \times 10^{38}$ so that in major computer systems with 24 bit mantissa whose maximum allowable floating point number is $3.4 \times 10^{38}$ overflow occurs if $|t| \geq 4.74$ in single precision computation. Watanabe's device is one of the methods to remedy the situation $[83,31]$.

Another problem is the loss of significant digits. If $f(x)$ has a singularity at the end point like $(1+x)^{-1+\mu}$ or $(1-x)^{-1+\mu}$ where $\mu$ is a small positive constant, we often encounter a large error due to the loss of significant digits at $x$ very close to -1 or 1 . In such a case we can avoid it by computing and storing the values

$$
\begin{aligned}
& \xi=x+1=\exp \left(\frac{\pi}{2} \sinh t\right) / \cosh \left(\frac{\pi}{2} \sinh t\right) \\
& \eta=1-x=\exp \left(-\frac{\pi}{2} \sinh t\right) / \cosh \left(\frac{\pi}{2} \sinh t\right)
\end{aligned}
$$

for $t=k h$ beforehand in addition to the values of the points $\phi(k h)$ and the weights $\phi^{\prime}(k h)$. Then, when we use the formula, we write a code of a function subprogram for $\tilde{f}(x, \xi, \eta)$ instead of one for $f(x)$. For example, if we want to integrate

$$
I=\int_{-1}^{1} f(x) d x, \quad f(x)=\frac{1}{(x-2)(1-x)^{1 / 4}(1+x)^{3 / 4}},
$$

we recommend to write a function subprogram for

$$
\tilde{f}(x, \xi, \eta)=\frac{1}{(x-2) \eta^{1 / 4} \xi^{3 / 4}}
$$

instead of one for $f$.

A careless coding results in the problems mentioned above and many people seem to have given up using the DE formula. This may be one of the reasons why the spread of the DE formula has not been so fast.

\section{§4.6. Publicity activities}

It may be said that after the DE transformation was established Takahasi and Mori were not so much eager to publicize it. Although Publ. RIMS is famous in the community of pure mathematicians it is not well-known among numerical analysts and their paper did not seem to attract people's attention in numerical analysis community so much.

In the mean time Mori had an opportunity to visit Courant Institute for Mathematical Sciences, New York University as a foreign visitor supported by 
the Ministry of Education, Japan. He stayed there for about one year from September 1974 until August 1975. In Courant Institute they had Numerical Analysis Seminar every Friday and regular members were P. D. Lax, F. John, E. Isaacson, L. Nirenberg, A. Jameson, O. B. Widlund, M. Shimasaki who was also visiting the Institute away from Kyoto University and Mori. Mori gave a talk there on February 281975 about the DE formula. For Mori it was the first time to give a talk outside Japan and a precious experience.

In March 1975 Gene Golub visited Courant Institute and Mori had an opportunity to tell Golub about the DE formula and to discuss about it with him. After Golub returned to Stanford University Mori received a phone call on May 12 from David Kahaner, Los Alamos, to invite him to Los Alamos Workshop on Quadrature Algorithms. Mori of course accepted the invitation and participated in the workshop. It turned out that the invitation was by Golub's suggestion. To Mori's regret he could not give a talk because the phone call from Kahaner was just three days before the opening day, May 15, so that it was too soon to change the program. But at this opportunity Mori got acquainted with many numerical analysts in the United States. Among them were P. J. Davis, W. Gautschi, G. H. Golub, F. G. Lether, J. Lyness, R. P. Moses, J. R. Rice, F. Stenger and J. B. Stroud. Just in this year P. J. Davis published the book "Methods of Numerical Integration" jointly with Philip Rabinowitz [4]. In the workshop Davis told Mori that their research result on the error estimation of numerical integration was cited in the book. Later Mori translated this book into Japanese and it was published in 1981 by Nippon Computer Kyokai.

At the end of Section 3 it was pointed out that when Takahasi and Mori submitted the paper [78] to Numerische Mathematik the referee brought the paper by C. Schwartz to their notice. The fact is that Frank Stenger was the referee which Stenger himself told Mori at the workshop. Stenger also told Mori that when he refereed their paper he was doing a similar research on the variable transformation [60]. Nevertheless his judgement was to accept it for publication. Since Mori heard about it he respects Stenger for his fairness.

\section{$\S 5 . \quad$ Developments of the DE Formula}

\section{§5.1. Numerical analysis group in Tsukuba}

In 1979 Mori moved to University of Tsukuba. He belonged to the Institute of Information Sciences and Electronics and, since it was a new university, he started to organize a numerical analysis group in cooperation with Yasuhiko 
Ikebe, Yoshio Oyanagi and Makoto Natori. Around that time Mori often participated in the annual meeting of Mathematical Society of Japan and every time he participated he was attracted by a talk given by a young researcher from University of Tokyo. His research subject was the good lattice points method to which he applied the DE transformation [71, 66]. His name was Masaaki Sugihara. Fortunately a vacant post was available at University of Tsukuba and in 1982 Sugihara left Tokyo to join the numerical analysis group in Tsukuba. Almost at the same time Kazuo Murota also moved to Tsukuba from University of Tokyo although the institute he belonged to was different.

In July 1975, just before Mori left New York to return to Kyoto Philip Rabinowitz from Israel dropped in at Courant Institute on his journey in the United States to meet Mori. At this opportunity Mori told Rabinowitz recent developments of the DE formula. In June 1980 Mori invited Rabinowitz to Tsukuba by JSPS program. On this occasion he also arranged a RIMS symposium on numerical integration. Takahasi participated in this symposium and met Rabinowitz. As a result Davis and Rabinowitz spared about one page for the DE formula in the second edition of their book "Methods of Numerical Integration" [4].

Mori was invited to give a talk at the first International Conference on Computational and Applied Mathematics at Leuven in July 1984 [27]. There Mori met Robert Piessens and they agreed to publish a special issue of Journal of Computational and Applied Mathematics on numerical integration. The special issue was published in 1987 in which the English translation [9] of the original paper [8] on the IMT-rule can be found.

Hidetosi Takahasi passed away in 1985. People lamented for his death.

Another unforgettable meeting for Mori was the International Congress of Mathematicians which was held in Kyoto. It was in 1990 just after he moved to the University of Tokyo as will be mentioned later. Mori was invited to give a talk on the DE formula there [31] and, although the audience was not so large, Mori thought it to be his great honor and felt that it was a commemorative event for the DE transformation.

\section{§5.2. Theorem of optimality of the DE formula by Sugihara}

As is mentioned at the end of Section 4, when Takahasi and Mori proposed the DE transformation Stenger was also investigating the optimality of quadrature formulas over $(-\infty, \infty)$ in a more mathematically rigorous manner [61]. His conclusion was that the optimal formula is the trapezoidal formula and its error behavior is $\exp (-c \sqrt{N})$ as a function of $N$ which differs from 
the result by Takahasi and Mori. It coincides with the error behavior by the single exponential transformation in [80]. Stenger who found the error behavior $\exp (-c N / \log N)$ in the paper by Takahasi and Mori [80] sent a letter to Mori claiming that the error behavior $\exp (-c N / \log N)$ is impossible and that accordingly their result is false. Indeed the reasoning by Takahasi and Mori was a little so intuitive as might have given some confusion to mathematicians.

Mori discussed with Sugihara and Murota about the letter from Stenger. After this discussion they concluded that the result by Stenger is true but that his claim was not relevant, i.e. the discrepancy is caused by the function space in which Stenger treated formulas. When Stenger discussed the optimal formula he established the theorem of optimality in a function space which consists of functions with single exponential decay [61]. On the other hand, Takahasi and Mori started from the class of functions with single exponential decay and narrowed argument down to the class of functions with double exponential decay although they did not use the term "function space".

In order for mathematicians to be convinced of the optimality of the DE formula Mori persuaded Sugihara to establish a theorem of optimality in a more mathematically rigorous manner because Mori knew that functional analysis is Sugihara's favorite subject and thought that it would be possible for Sugihara to bring it successful.

Answering Mori's expectation Sugihara soon challenged this problem from his own stand point. Sugihara's idea can be summarized as follows. He first investigated the optimality in the function space whose elements decay single exponentially. Next he investigated it in the function space whose elements decay double exponentially. Then he found that the trapezoidal formula is optimal in each of both function spaces and that the error of the trapezoidal formula approaches zero faster in the function space with double exponential decay than in the space with single one as the number of function evaluations increases. Then, in order to find a more efficient transformation, it is natural to consider next a function space whose elements decay faster than double exponentially. To this problem he gave a negative answer by proving a theorem that there exists no function that decays faster than double exponentially with an exponent $\pi /(2 d)$ as $\operatorname{Re} w \rightarrow \pm \infty$ as long as we think of functions regular in the strip region $|\operatorname{Im} w|<d$. Thus Sugihara succeeded in establishing a theorem for the optimality of the trapezoidal formula combined with the double exponential decay of integrands, although the conclusion was on the whole the same as that by Takahasi and Mori. Incidentally it should be noted that Sugihara's non-existence theorem stated above seems to contradict the analysis 
by Takahasi and Mori in that they considered functions whose decay is faster than double exponential. But it is not the case because they could do it since they allowed the singularities of the weight function $\phi^{\prime}(w)$ to approach to the real axis as $\operatorname{Re} w \rightarrow \pm \infty$.

Sugihara first presented the result orally at a RIMS symposium in 1986 [64] and it took more than ten years for him until he completed and published his theorem in 1997 in Numerische Mathematik [67]. Mori thinks that the reason why it took more than ten years for Sugihara to complete the work would be that he is very careful and prudent for everything.

At the end of this subsection the author would like to give a comment on the significance of the DE transformation. There may be mathematicians who say that, since the space of functions with single exponential decay includes the space of functions with double exponential decay, the DE formula may be regarded, without mentioning its merit explicitly, as merely a part of the result of analysis in the function space with single exponential decay. It is logically true. But the author has a different opinion. From the view point of applied mathematicians H. Takahasi and M. Mori were aiming from the beginning at finding a transformation that leads to a useful formula which gives a result of the highest precision with the minimum number of function evaluations. And hence the family of functions with double exponential decay has a special significance for them while the family of functions with single exponential decay is just a temporary test family in the process of their analysis.

\section{$\S 5.3 . \quad$ Books by Mori}

While Mori was working in RIMS he wrote several books on numerical analysis and related topics. These books were written in Japanese. He made all possible effort when he wrote "Numerical Analysis" published in 1973 by Kyoritsu Shuppan [20] because it was his first book. However, unfortunately there was no description about the DE formula because it was published just one year before the paper [80] was published. It sold well but later around 1990 it went out of print. However, in 2002 Kyoritsu Shuppan changed their mind to republish it in the second edition. At this opportunity Mori rewrote it partially and added a few pages for the DE formula. Incidentally, details of the DE formula can be found in his "Complex Function Theory and Numerical Analysis" published in 1975 by Chikuma Shobo [23].

In order to plot contour maps of the characteristic functions of the error Mori wrote many programs in Fortran. He collected them in his book "Curves and Surfaces" which was published in 1974 by Kyoiku Shuppan [22] and in 
a later one "Graph Processing Methods and FORTRAN 77 Programming" in 1991 by Iwanami Shoten [32].

Since Mori has been interested not only in numerical integration but also in other methods of numerical computation in general, he also wrote a great deal of programs of library subroutines in Fortran. He collected these subroutines in "Numerical Methods and FORTRAN 77 Programming" published in 1991 by Iwanami Shoten [28].

In this book a several DE formulas in the form of automatic integrator are included. In particular in the second edition a subroutine of the DE formula for integrals $\int_{0}^{\infty} f_{1}(x) d x$ given in (4.16) was included. After the subroutine program Mori gave a comment that this DE formula does not work well if the integrand is an oscillatory slowly decaying function like

$$
I=\int_{0}^{\infty} \frac{1}{1+x^{2}} \sin x d x .
$$

This is because if the transformation $z=\exp (\pi / 2 \sinh w)$ is applied the transformed integrand has an infinite array of singularities which approach to the real axis as Re $w \rightarrow \pm \infty$. In $1978 \mathrm{H}$. Toda and H. Ono [82] and later in 1987 Sugihara [65] used a Richardson's exterplation in order to overcome this drawback.

\section{§5.4. Ooura's transformation for oscillatory integrals}

In April 1989 Mori moved to University of Tokyo. The institute he belonged to was Department of Applied Physics, the same place as he previously worked for. Soon after Mori's transfer to Tokyo Sugihara also moved to join Mori at University of Tokyo.

One day in June 1989 Mori received a letter from a student. He began his letter saying "I am a second year undergraduate student from Nagoya University ...". He continued that he read Mori's book [28] and that he found a transformation by which oscillatory integrals with a slowly decaying integrand like (5.1) can be evaluated efficiently. Mori read the letter half in doubt but carefully and found that what the student said was completely correct. His name is Takuya Ooura.

Ooura's idea is as follows. Suppose that we want to evaluate an integral

$$
I=\int_{0}^{\infty} f_{1}(x) \sin \omega x d x
$$

where $f_{1}(x)$ is a slowly decaying algebraic function. For this integral Ooura 
proposed a transformation

$$
x=M \phi(t), \quad \phi(t)=\frac{t}{1-\exp (-6 \sinh t)} .
$$

$M$ should be chosen as will be mentioned below. If we carry out variable transformation (5.3) to (5.2) and apply the trapezoidal formula we have

$$
I_{h}=M h \sum_{k=-\infty}^{\infty} f_{1}(M \phi(k h)) \sin (M \omega \phi(k h)) \phi^{\prime}(k h),
$$

where we must choose $M$ and $h$ in such a way that

$$
M \omega h=\pi
$$

holds. Note that for large negative $k h$ the integrand decays double exponentially from (5.3). On the other hand for large positive $k h$, although the integrand does not decay at all,

$$
\sin (\omega M \phi(k h)) \approx \sin M \omega k h=\sin k \pi=0
$$

holds since $\phi(t) \rightarrow t$ for large positive $t$ so that the point of the formula approaches to the zero of $\sin \omega M \phi(t)$. Therefore we do not have to evaluate the integrand for large positive $k h$ and can truncate the infinite summation at a moderate value of $k$.

As an example we consider

$$
I=\int_{0}^{\infty} \log x \sin x d x=-\gamma=-0.57721566490153 \cdots
$$

where $\gamma$ is the Euler's constant. The logarithmic term $\log x$ in (5.7) increases slowly as $x$ becomes large and such kind of an integral should be defined

$$
I=\lim _{\varepsilon \rightarrow+0} \int_{0}^{\infty} e^{-\varepsilon x} \log x \sin x d x=-\gamma .
$$

In Fig.5 the graph of the integrand $\log x \sin x$ is shown. Short dashes indicate the location of the points of the formula and we see that they approach to the zeros of the integrand as $x$ becomes large. If we apply (5.3) with $M=50$ we obtain an approximate value of $-\gamma$ whose absolute error is about $2.1 \times 10^{-13}$ with $N=75$ function evaluations.

Mori re-examined Ooura's analysis and carried out various sorts of numerical integration with Ooura's transformation and Mori, under Ooura's agreement, gave a joint talk at a RIMS symposium in November 1989. Their paper was published in 1991 [53]. 


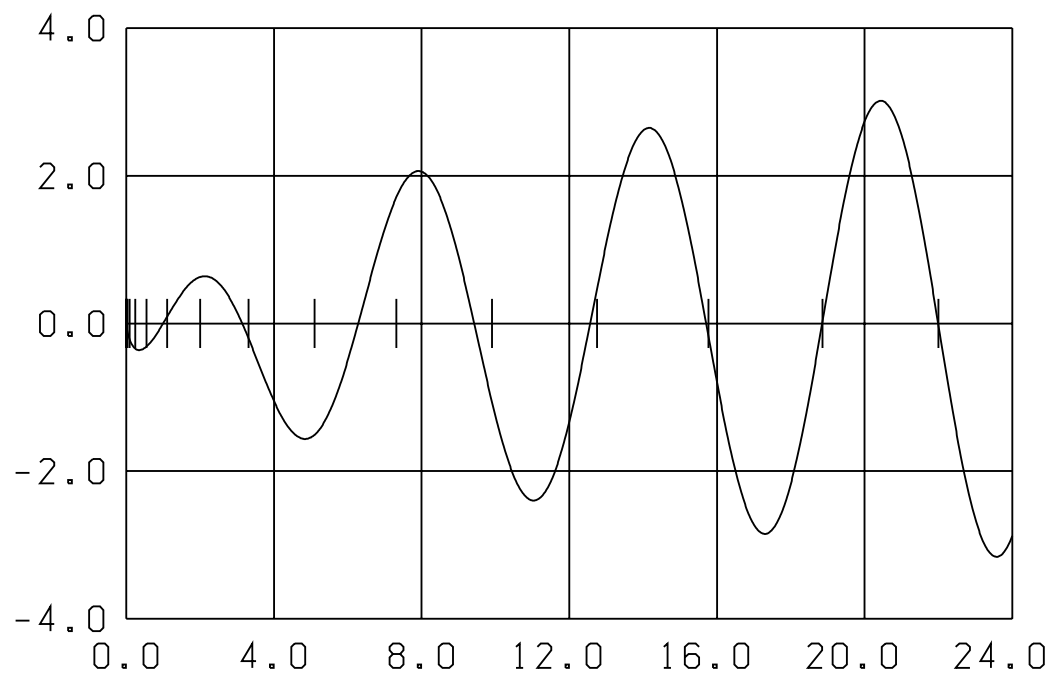

Figure 5. Graph of $\log x \sin x$

After Ooura graduated from Nagoya University in 1992 he entered the graduate course in the University of Tokyo and obtained the doctor degree in 1997 [52] under the supervision of Mori. He is now working for RIMS as a research associate and is still giving excellent results in the field of numerical analysis $[54,50,55]$ in which the continuous Euler's transformation should deserve special mention $[49,51]$.

\section{§5.5. Permeation of the DE transformation}

In the University of Tokyo good students entered the laboratory of Mori and Sugihara. Ooura is one of them. Sometimes they worked together [45] and sometimes independently. Hidenori Ogata investigated formulas for Cauchy principal-value integrals and for Hadamard finite-part integrals based on the DE transformation [48]. He also proposed DE formulas for integrals involving the Bessel functions [46, 47]. Ogata was Sugihara's student and hence many of his papers are joint ones with Sugihara. Takayasu Matsuo, also one of Sugihara's students, applied the DE method to a sinc-type pseudospectral method [16].

Since the DE formula was proposed in 1974 it spread slowly but steadily among people who are actually working in the fields of numerical computation. 
At the beginning it was confined inside Japan. In almost all the subroutine libraries at computer centers of Japanese universities subroutines for the DE formula could be found already around 1985. DE subroutines in NUMPACK originally from Nagoya University is still provided for public use. Subroutines using the DE formula were also developed by representative computer makers in Japan. Library packages for supercomputers from Hitachi, NEC and Fujitsu included several subroutines of the DE formulas at the time of around 1990.

One of the typical examples in which application of the DE formula was successful is a paper by Momose et al. in 1987. They used the DE formula for evaluation of molecular integrals [18]. Use of the DE formula is still spreading. In fact, K. Kobayashi and H. Okamoto devised a combination of the DE transform with the Fourier transform and gave an interesting result in the field of fluid dynamics [10] in 2003. Recently J. W. Maina and K. Matsui applied the DE formula to a problem encountered in civil engineering [14]. Also, Y. Yamamoto devised a method which improves remarkably the efficiency of computation appearing in a financial problem [84].

The talk given by Mori at the first International Conference on Computational and Applied Mathematics at Leuven in July 1984 [27] and the one at the International Conference on Numerical Mathematics, Singapore in June 1988 [29], and also the description in the second edition of the book by Davis and Rabinowitz published in 1984 [4] seem to have contributed to the spread of the DE formula outside Japan. Actually we find a small citation of the DE formula in the book by H. R. Schwartz published in 1989 [59] and a full description in the book by S. Nakamura published in 1991 [42], for example. In QUADPACK the DE formula is not included although it is cited in [56].

In September 1996 Mori gave a lecture on the optimality of the DE transform at the 50th anniversary of the foundation of Mathematical Society of Japan $[33,34]$. This is an example of scarce opportunities for Mori to present his research results on the DE transformation to mathematicians in Japan.

In 1997 Mori was awarded the 28th Ishikawa Prize for the discovery of the double exponential transformation.

The DE formula seems to have been used most successfully in the boundary element method (BEM). As far as the author knows the first paper on the application to BEM is the one by T. Higashimachi et al. in 1983 in the field of computational mechanics [6]. In 1995 N. Kunihiro et al. devised an efficient composition of variable transformations including the DE transformation in BEM [12]. In order to give here the present status in BEM we quote a comment in a paper by A. Aimi et al. [2]: 
For 2D integration of smooth functions except at the end points where they may have log-singularities we sugest to use the DE-rule, which has been widely and successfully applied in 2D problems.

\section{§6. DE Sinc Methods and Further Developments}

\section{§6.1. Sinc expansion}

It is said [72] that a function defined by

$$
\operatorname{sinc}(x)=\frac{\sin \pi x}{\pi x}
$$

has been playing an important role in the field of information theory. If $g(w)$ is a function regular in the strip region $|\operatorname{Im} w|<d$ for some fixed $d$ it can be expanded

$$
g(t)=\sum_{k=-\infty}^{\infty} g(k h) \operatorname{sinc}\left(\frac{t}{h}-k\right)+e(t, h, d)
$$

where $e(t, h, d)$ is the error term. This expansion is called the sinc expansion. While in the western literatures on numerical analysis we see the derivation of (6.2) rather later [63], in Japan Hidetosi Takahasi mentioned the importance and the usefulness of the sinc expansion already in his lecture at a RIMS symposium in 1975 [72] although he did not use the term "sinc". In fact he started his lecture with the identity

$$
g(t)-\hat{g}(t)=\frac{\sin \frac{\pi t}{h}}{2 \pi i} \int_{C} \frac{g(w)}{(w-t) \sin \frac{\pi w}{h}} d w(=e(t, h, d))
$$

where $\hat{g}(t)$ is defined

$$
\hat{g}(t)=\sum_{k=-\infty}^{\infty} g(k h) \operatorname{sinc}\left(\frac{t}{h}-k\right)
$$

and the contour $C$ surrounds the point $w=t$ and all the zeros of $\sin (\pi w / h)$. If we apply the residue theorem to the integral in the right hand side of (6.3) we immediately get (6.2). Incidentally, we should note that the following relation between the sinc expansion and the trapezoidal sum can easily be proved:

$$
\int_{-\infty}^{\infty} \hat{g}(t) d u=h \sum_{k=-\infty}^{\infty} g(k h)
$$


Also note that $\hat{g}(t)$ is an expansion of convolution type. Takahasi called the function sinc $(t / h)$ in (6.4) the Shannon's interpolant since $\hat{g}(t)$ satisfies $\hat{g}(k h)=$ $g(k h)$. He pointed out that if $g(w)=o(\exp (\pi|\operatorname{Im} w| / h))$ holds for large $|\operatorname{Im} w|$ then $\hat{g}(t)=g(t)$ holds exactly. Also Takahasi proposed a method to accelerate the convergence of the series (6.4).

To Mori's regret he missed Takahasi's lecture at RIMS in 1975 because Mori was visiting Courant Institute just at that time and hence he did not notice the lecture note [72] at the moment. Thus no body followed Takahasi's suggestion for sinc expansion until several years later. However, in order to pulicize Takahasi's idea among people outside Japan, Mori, in cooperation with H. Okamoto and M. Sugihara, translated Takahasi's lecture note [72] into English. It is going to be published in the present issue [73].

In any case the sinc expansion (6.2) plays a central role in the following discussion. For these ten years research activities in the field of sinc expansion have been flourished by Frank Stenger [63] and people in his school. However, the transformations they used were of single exponential type and the resulting convergence rate was again $\exp (-C \sqrt{N})$ where $N$ is the number of terms of the sinc expansion.

On the other hand, Sugihara thought that the DE transformation is also useful in the sinc approximation [70]. He and his students successfully applied the sinc method based on the DE transformation to two-point boundary value problems of second order differential equation [7, 68] and to Sturm-Liouville type eigenvalue problems [11].

In 2003 Sugihara established a theorem on a near optimality of the sinc approximation incorporated with the DE transformation based on a similar idea as in the optimality of the DE formula for definite integrals [69].

In the meantime Mori retired from the University of Tokyo at the age of 60. Just after the retirement he moved to RIMS, Kyoto University and there he hold the position of the director for three years from 1998 until 2001. There he spent a useful time with his former students Takuya Ooura and Daisuke Furihata because these two persons also were working for RIMS at the same time. Furihata's research subject was different from the DE transform, i.e. the discrete variation which he developed in the University of Tokyo under Mori's supervision before he moved to RIMS. Although Mori was occupied by the business as the director he could charge various new ideas around the DE transform through discussions with these young researchers. 


\section{§6.2. Indefinite DE integration}

In 2001 Mori retired from RIMS, Kyoto University at the age of 63 and moved to Tokyo Denki University, a private university near Tokyo. There he was again blessed with a student, Mayinur Muhammad from Uighur, China. Fortunately her husband Ahniyaz Nurmuhammad who is also working for Tokyo Denki University is a mathematician and his cooperation has been very helpful for Mori and Muhammad. Mori and his co-researchers extensively applied the sinc method based on the DE transformation to various kinds of numerical problems in accordance with Sugihara's suggestion. First, Mori and Muhammad applied the DE transformation to indefinite integration [35, 37]. Application of the sinc method to indefinite integration had been already investigated by F. Stenger [62] and later by S. Haber [5] although they used mainly the transformation of single exponential type. Mori and Muhammad followed their analysis by replacing the single exponential transformation with the double exponential one. Suppose that we want to evaluate an indefinite integral

$$
I(s)=\int_{-1}^{s} f(x) d x, \quad-1<s<1 .
$$

Although they assumed that $f(x)$ is expressed $f(x)=\left(1-x^{2}\right)^{-\alpha} f_{1}(x), \alpha<1$ we will assume here $\alpha=0$ for simplicity as in the analysis in Section 3 and Section 4. We apply the DE transformation

$$
x=\phi(t)=\tanh \left(\frac{\pi}{2} \sinh t\right)
$$

to the indefinite integral and subsequently apply the trapezoidal formula with mesh size $h$ to obtain

$$
\begin{aligned}
I(s)=\int_{-\infty}^{\tau} f(\phi(t)) \phi^{\prime}(t) d t= & h \sum_{k=-\infty}^{\infty} f(\phi(k h)) \phi^{\prime}(k h)\left(\frac{1}{2}+\frac{1}{\pi} \operatorname{Si}\left(\pi \frac{\tau}{h}-\pi k\right)\right) \\
& +O\left(h \exp \left(-\frac{\pi d}{h}\right)\right), \quad \tau=\phi^{-1}(s)
\end{aligned}
$$

where $\operatorname{Si}(x)$ is the sine integral defined by

$$
\operatorname{Si}(x)=\int_{0}^{x} \frac{\sin \xi}{\xi} d \xi .
$$


If we truncate the infinite summation at $k= \pm n$ according to the criterion (3.18) we have

$$
h=\frac{2}{N} \log d N, \quad N=2 n+1
$$

and eventually obtain the DE formula for indefinite integration

$$
\begin{aligned}
\int_{-1}^{s} f(x) d x= & h \sum_{k=-\infty}^{\infty} f(\phi(k h)) \phi^{\prime}(k h)\left(\frac{1}{2}+\frac{1}{\pi} \operatorname{Si}\left(\pi \frac{\phi^{-1}(s)}{h}-\pi k\right)\right) \\
& +O\left(\exp \left(-\frac{\pi d N}{2 \log (d N)}\right)\right) .
\end{aligned}
$$

We call $x_{k}=\phi(k h), k=-n,-n+1, \ldots, n$ the sinc points. The single exponential transformation gives a formula with an error term

$$
O\left(N^{1 / 2} \exp \left(-(\pi d N / 2)^{1 / 2}\right)\right), \quad h=(2 \pi d / N)^{1 / 2} .
$$

Incidentally it should be noted that an independent work on the indefinite DE integration is given by Tanaka jointly with Sugihara and Murota [81].

The role of the function $\left(1 / 2+1 / \pi \operatorname{Si}\left(\pi \phi^{-1}(s) / h-\pi k\right)\right)$ appearing in the right hand side of (6.8) is interesting. This is the main difference between the formula for the definite integral (3.12) and the one for the indefinite integral (6.8). Consider here a function defined by

$$
Y_{h, \tau}(t)=\frac{1}{2}+\frac{1}{\pi} \operatorname{Si}\left(\frac{\pi}{h}(\tau-t)\right), \quad \tau=\phi^{-1}(s) .
$$

If we integrate $f(\phi(t)) \phi^{\prime}(t) Y_{h, \tau}(t)$ over $(-\infty, \infty)$ by the trapezoidal formula with mesh size $h$, then we have the formula (6.11). In Fig.6 we show the graph of $Y_{h, \tau}(t)$ with $h=0.25$ and $\tau=0$. This graph indicates that $Y_{h, \tau}(t)$ approximately cuts off the function $f(\phi(t)) \phi^{\prime}(t)$ at $t=\tau$ in (6.8) which corresponds to the cut off of the integration of (6.6) at the upper bound $x=s$. In this sense $Y_{h, \tau}(t)$ plays a role an approximation to the discontinous Heaviside step function. Although $Y_{h, \tau}(t)$ is an approximation $f(\phi(t)) \phi^{\prime}(t) Y_{h, \tau}(t)$ is regular in the strip region $|\operatorname{Im} w|<d$, so that its integration gives again a result with very high accuracy.

\section{§6.3. Applications of indefinite DE integration}

As a direct application of the formula for indefinite integrals Muhammad and Mori considered numerical evaluation of an iterated integral

$$
I=\int_{a}^{b} d x \int_{a^{\prime}}^{q(x)} f(x, y) d y
$$




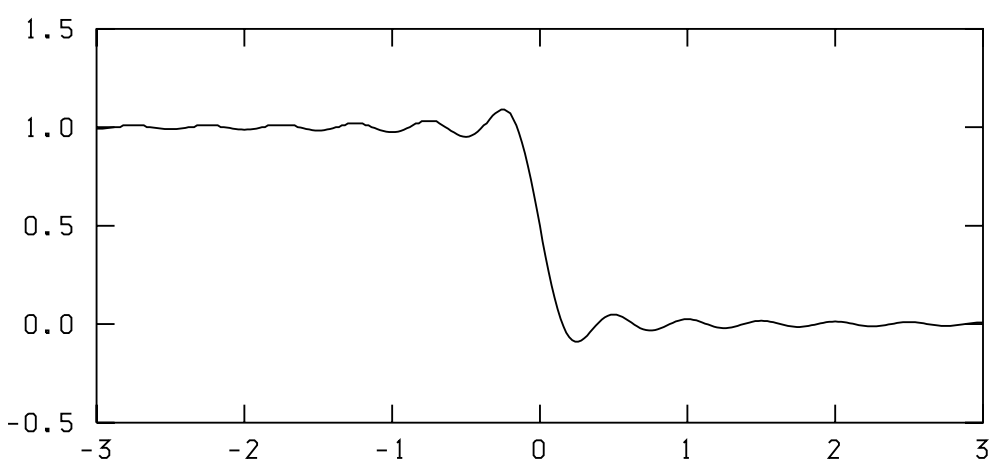

Figure 6. $Y_{h, \tau}(t)=\frac{1}{2}+\frac{1}{\pi} \mathrm{Si}\left(\frac{\pi}{h}(\tau-t)\right), h=0.25, \tau=0$

where $q(x)$ is an increasing function satisfying

$$
q(a)=a^{\prime}, \quad q(b)=b^{\prime} \quad(a<x<b) .
$$

They applied the indefinite DE formula (6.11) to the inner indefinite integration in (6.14) and then subsequently applied the conventional definite DE formula (4.11) to the outer definite integration. The result was as expected and satisfactory $[36,38]$. If the integrand is of a product type, i.e. $f(x, y)=X(x) Y(y)$, it reduces to essentially a one dimensional numerical integration.

Muhammad and her co-researchers applied the DE transformation to numerical solution of a Volterra integral equation of the second kind

$$
u(x)-\lambda \int_{a}^{x} K(x, u(\xi)) d \xi=g(x), \quad a<x<b
$$

where the constant $\lambda$ and the function $g(x)$ are given and $u(x)$ needs to be found. They approximated the kernel integral in (6.16) using the indefinite DE integration stated above and subsequently applied collocation based on the sinc points $x_{k}=\phi(k h), k=-n,-n+1, \ldots, n$. In this way they obtained an efficient formula to solve (6.16) [39]. If $K(x, u)$ is linear with respect to $u$, the resulting system of algebraic equations can be directly solved by means of the Gauss elimination. If $K(x, u)$ is nonlinear, on the other hand, we need some iterative method, say Newton's iteration. 
Suppose that we want to solve an initial value problem of ordinary differential equation

$$
\left\{\begin{array}{l}
\frac{d u}{d x}=K(x, u), \quad a<x<b \\
u(a)=u_{0} .
\end{array}\right.
$$

If we integrate (6.17) with respect to $x$ under the given boundary condition we immediately have the Volterra integral equation (6.16). Then, using the method given above we obtain an approximate solution of the original initial value problem [44]. They observed that it works well also for stiff problems without any special care for the stiffness.

There had been several researches on numerical solution of Volterra integral equation and, consequently, initial value problem of ordinary differential equation based on the single exponential transformation which preceded their work [63]. However, here also they brought the DE transformation into focus and obtained significant results.

Recently Nurmuhammad and his co-researchers jointly with Sugihara applied the sinc collocation method successfully to boundary value problems of fourth order differential equation [43].

On the other hand Stenger and people in his school are developing and enhancing a package "Sinc-Pack" using the DE transformation. The author puts his hopes on a friendly competition between Stenger's group and the DE group in Japan.

\section{$\S 7 . \quad$ Conclusions}

The author would like to point out as the first concluding remark that the DE transformation is one of the not many academic harvests in the field of applied mathematics that were discovered and developed inside Japan and spread out worldwide.

Applications of the DE transformation to evaluation of definite integrals are still permeating in various fields of science and engineering as pointed out at the end of Section 5. Recently the DE formula is incorporated into well known mathematical software such as Maple and Mathematica. Visit the web-page of Mathworld [15] for example.

Applications of the indefinite DE integration are just beginning as mentioned in the previous section. Since it can be used for solution of integral equations and also for solution of differential equations the author hopes that the scope of its applications will go beyond expectation. 
As the last concluding remark we cite here a comment from the report by D. H. Bailey and X. S. Li in 2003 on a comparison of high-precision quadrature schemes [3]:

The tanh-sinh $(=\mathrm{DE})$ scheme appears to be the best. It combines uniformly excellent accuracy with fast run times. It is the nearest we have to a truly all-purpose quadrature scheme at the present time.

Finally the author would like to express his sincere gratitude to Professor Hisashi Okamoto for his perpetual kind support to disseminate the DE transforms. In particular the workshop "Thirty Years of the Double Exponential Transforms" and this special issue would not have been realized without his effort. Also, the author would like to thank the referees for giving valuable comments.

\section{References}

[1] Aihie, V. U. and Evans, G. A., A comparison of the error function and the tanh transformation as progressive rules for double and triple singular integrals, J. Comput. Appl. Math., 30 (1990), 145-154.

[2] Aimi, A. and Diligenti, M., Hypersingular kernel integration in 3D Galerkin boundary element method, J. Comput. Appl. Math., 138 (2002), 51-72.

[3] Bailey, D. H. and Li, X. S., A comparison of three high-precision quadrature schemes, Proceedings of the 5th Conference on Real Numbers and Computers, September 3-5, 2003, Ecole normale superieure de Lyon, Lyon, France.

[4] Davis, P. J. and Rabinowitz, P., Methods of Numerical Integration, Academic Press, 1st edition 1975, 2nd edition 1984, Japanese translation by M. Mori, Nippon Computer Kyokai, 1981.

[5] Haber, S., Two formulas for numerical indefinite integration, Math. Comp., 60 (1993), 279-296.

[6] Higashimachi, T., Okamoto, N., Ezawa, Y., Aizawa, T. and Ito, A., Interactive structural analysis system using the advanced boundary element method, Proceedings of the Fifth International Conference on Boundary Elements, Hiroshima, November 1983, Eds. Brebbia, C. A., Futagami, T. and Tanaka, M., A Computational Mechanics Center Publication, Springer-Verlag, 1983, 847-856.

[7] Horiuchi, K. and Sugihara, M., Sinc-Galerkin method with the double exponential transformation for the two point boundary problems, Tech. Rep., 99-05, Dept. of Mathematical Engineering, University of Tokyo, 1999.

[8] Iri, M., Moriguti, S. and Takasawa, Y., On a certain quadrature formula, (in Japanese), Kokyuroku RIMS, Kyoto Univ., 91 (1970), 82-119.

[9] $\longrightarrow$, On a certain quadrature formula, J. Comput. Appl. Math., 17 (1987), 3-20 (translation of the original paper in Japanese [8]).

[10] Kobayashi, K., Okamoto, H. and Zhu, J., Numerical computation of water and solitary waves by the double exponential transform, J. Comp. Appl. Math., 152 (2003), 229-241.

[11] Koshihara, T. and Sugihara, M., A numerical solution for the Sturm-Liouville type eigenvalue problems employing the double exponential transformation (in Japanese), Proceedings of 1986 Annual Meeting of the Japan Society for Industrial and Applied Mathematics, 1986, 136-137.

[12] Kunihiro, N., Hayami, K. and Sugihara, M., Automatic numerical integration for the boundary element method using variable transformation and its error analysis (in Japanese), Trans. Japan Soc. Industr. Appl. Math., 5 (1995), 101-119. 
[13] Lund, J. and Bowers, K. L., Sinc Methods for Quadrature and Differential Equations, SIAM, 1992.

[14] Maina, J. W. and Matsui, K., Development of software for elastic analysis of pavement structure due to vertical and horizontal surface loadings, Transportation Research Board 2004 Annual Meeting, to appear in J. Transportation Research Board.

[15] Mathworld: http://mathworld.wolfram.com/DoubleExponentialIntegration.html

[16] Matsuo, T., On an application of the DE transformation to a Sinc-type pseudospectral method (in Japanese), Proceedings of 1997 Annual Meeting of the Japan Society for Industrial and Applied Mathematics, 1997, 36-37.

[17] McNamee, J., Eror bounds for the evaluation of integrals by the Euler-Maclaurin formula and by Gauss-type formulae, Math. Comp., 18 (1964), 368-381.

[18] Momose, T. and Shida, T., Efficient formulas for molecular integrals over the HillerSucher-Feinberg identity using Cartesian Gaussian functions: Towards the improvement of spin density calculation, J. Chem. Phys., 87 (1987), 2832-2846.

[19] Mori, M., Numerical analysis and theory of hyperfunction (in Japanese), Kokyuroku RIMS, Kyoto Univ., 145 (1972), 1-11.

[20] , Numerical Analysis (in Japanese), Kyoritsu Shuppan, Tokyo, 1973, 2002.

[21] On the superiority of the trapezoidal rule for the integration of periodic analytic functions, Memoirs of Numerical Mathematics, 1 (1974), 11-19.

[22] Curves and Surfaces (in Japanese), Kyoiku Shuppan, Tokyo, 1974.

[23] Complex Function Theory and Numerical Analysis (in Japanese), Chikuma Shobo, Tokyo, 1975.

[24] Japanese), Kokyuroku, RIMS, Kyoto Univ., 310 (1977) 32-47.

[25] - An IMT-type double exponential formula for numerical integration, Publ. RIMS, Kyoto Univ., 14 (1978), 713-729.

[26] A method for evaluation of the error function of real and complex variable with high relative accuracy, Publ. RIMS, Kyoto Univ., 19 (1983), 1081-1094.

[27] $\quad$ Quadrature formulas obtained by variable transformation and the DE-rule, $J$. Comput. Appl. Math., 12 \& 13 (1985), 119-130.

[28] _ Numerical Methods and FORTRAN 77 Programming (in Japanese), Iwanami Shoten, Tokyo, 1986, 168-186.

[29] The double exponential formula for numerical integration over the half infinite interval, in: Numerical Mathematics Singapore 1988, International Series of Numerical Mathematics 86, Birkhäuser, Basel, 1988, 367-379.

[30] - An error analysis of quadrature formulas obtained by variable transformation, in: M. Kashiwara and T. Kawai, eds., Algebraic Analysis Vol.1, Academic Press, Boston, 1988, 423-437.

[31] Developments in the double exponential formulas for numerical integration, Proceedings of the International Congress of Mathematicians, Kyoto 1990, SpringerVerlag, Tokyo, 1991, 1585-1594.

[32] - Graph Processing Methods and FORTRAN 77 Programming (in Japanese), Iwanami Shoten, Tokyo, 1991

[33] Optimality of the double exponential transformation in numerical analysis (in Japanese), Sūgaku, 50 (1998), 248-264.

[34] , Optimality of the double exponential transformation in numerical analysis, Sugaku Expositions, 14 (2001), 103-123. (In this article Fig.3 and Fig.4 should be reversed.) (translation of the original paper in Japanese [33]).

[35] Mori, M. and Muhammad, M., Numerical indefinite integration by the double exponential transformation (in Japanese), Trans. Japan Soc. Industr. Appl. Math., 13 (2003), 361-366.

[36] - Numerical iterated integration by the double exponential transformation (in Japanese), Trans. Japan Soc. Industr. Appl. Math., 13 (2003), 485-493. 
[37] Muhammad, M. and Mori, M., Double exponential formulas for numerical indefinite integration, J. Comput. Appl. Math., 161 (2003), 431-448.

[38] - Numerical iterated integration based on the double exponential transformation, Japanese J. Industr. Appl. Math., 22 (2005), 77-86.

[39] Muhammad, M., Nurmuhammad, A., Mori, M. and Sugihara, M., Numerical solution of integral equations by means of the Sinc collocation method based on the double exponential transformation, J. Comput. Appl. Math., 177 (2005), 269-286.

[40] Murota, K., On numerical quadrature formulas robust against endpoint singularities, Graduation Thesis, University of Tokyo, 1978.

[41] Murota, K. and Iri, M., Parameter tuning and repeated application of the IMT-type transformation in numerical quadrature, Numer. Math., 38 (1982), 327-363.

[42] Nakamura, S., Applied Numerical Methods with Software, Prentice-Hall Inc., 1991, p.132.

[43] Nurmuhammad, A., Muhammad, M., Mori, M. and Sugihara, M., Double exponential transformation in the Sinc-collocation method for a boundary value problem of fourthorder ordinary differential equation, J. Comput. Appl. Math., 182 (2005), 32-50.

[44] - Numerical solution of initial value problems of ODE based on the double exponential transformation, to appear in Publ. RIMS, Kyoto Univ., the present issue (2005).

[45] Ogata, H. and Ooura, T., Theoretical error analysis of a DE-type quadrature formula for oscillatory integrals (in Japanese), Proceedings of 1997 Annual Meeting of the Japan Society for Industrial and Applied Mathematics, 1997, 18-19.

[46] Ogata, H. and Sugihara, M., Interpolation and quadrature formulae whose abscissae are the zeros of the Bessel functions (in Japanese), Trans. Japan Soc. Industr. Appl. Math., 6 (1996), 39-66.

[47] _ Quadrature formulae for oscillatory infinite integrals involving the Bessel functions (in Japanese), Trans. Japan Soc. Industr. Appl. Math., 8 (1998), 223-256.

[48] Ogata, H., Sugihara, M. and Mori, M., A DE-type quadrature rule for Cauchy principalvalue integrals and Hadamard finite-part integrals (in Japanese), Trans. Japan Soc. Industr. Appl. Math., 3 (1993), 309-322.

[49] Ooura, T., An extension of the Euler transformation (in Japanese), Proceedings of 1993 Annual Meeting of the Japan Society for Industrial and Applied Mathematics, 1993, 111-112.

[50] - A new variable transformation of Fourier-type integrals (in Japanese), Proceedings of 1994 Annual Meeting of the Japan Society for Industrial and Applied Mathematics, 1994, 260-261.

[51] , A method of computation of oscillatory integrals using a continuous Euler transformation and the DE transformation (in Japanese), Proceedings of 1996 Annual Meeting of the Japan Society for Industrial and Applied Mathematics, 1996, 22-23.

[52] Study on numerical integration of Fourier type integrals (in Japanese), Doctoral Thesis, University of Tokyo, 1997.

[53] Ooura, T. and Mori, M., The double exponential formula for oscillatory functions over the half infinite interval, J. Comput. Appl. Math., 38 (1991), 353-360.

[54] - Double exponential formula for Fourier type integrals with a divergent integrand, in: Contributions in Numerical Mathematics, World Scientific Series in Applicable Analysis, 2 (1993), 301-308.

[55] _ A robust double exponential formula for Fourier type integrals, J. Comput. Appl. Math., 112 (1999), 229-241.

[56] Piessens, R., de Doncker-Kapenga, E., Überhuber, C. W., Kahaner, D. K., QUADPACK - A Subroutine Package for Automatic Integration, Springer-Verlag, Berlin, 1983.

[57] Sato, M., Theory of hyperfunctions I, J. Fac. Sci. Univ. Tokyo, 8 (1959), 139-193.

[58] Schwartz, C., Numerical integration of analytic functions, J. Comput. Phys., 4 (1969), 19-29.

[59] Schwartz, H. R., Numerical Analysis, A Comprehensive Introduction, John-Wiley \& Sons Ltd., 1989, p350. 
[60] Stenger, F., Integration formulas based on the trapezoidal formula, J. Inst. Math. Appl., 12 (1973), 103-114.

[61] - Optimal convergence of minimum norm approximation in $H_{p}$, Numer. Math., 29 (1978), 345-362.

[62] , Numerical methods based on the Whittaker cardinal, or sinc functions, SIAM Rev., 23 (1981), 165-224.

[63] Stenger, F., Numerical Methods Based on Sinc and Analytic Functions, Springer-Verlag, New York, 1993.

[64] Sugihara, M., Optimality of the double exponential formula, Kokyuroku RIMS, Kyoto Univ., 585 (1986), 150-175.

[65] - Methods of numerical integration of oscillatory functions by the DE-formula with the Richardson extrapolation, J. Comput. Appl. Math., 17 (1987), 47-68.

[66] Method of good matrices for multi-dimensional numerical integrations — An extension of the method of good lattice points, J. Comput. Appl. Math., 17 (1987), 197-213.

[67] _ Optimality of the double exponential formula — functional analysis approach — , Numer. Math., 75 (1997), 379-395.

[68] Double exponential transformation in the Sinc-collocation method for two-point boundary value problems, J. Comput. Appl. Math., 149 (2002), 239-250.

[69] Near optimality of the sinc approximation, Math. Comp., 72 (2003), 767-786.

[70] Sugihara, M. and Matsuo, T., Recent developments of the Sinc numerical methods, J. Comput. Appl. Math., 164/165 (2004), 673-689.

[71] Sugihara, M. and Murota, K., A note on Haselgrove's method for numerical integration, Math. Comp., 39 (1982), 549-554.

[72] Takahasi, H., Complex function theory and numerical analysis (in Japanese), Kokyuroku RIMS, Kyoto Univ., 253 (1975), 24-37.

[73] Complex function theory and numerical analysis, to appear in Publ. RIMS, Kyoto Univ., the present issue (2005). (translation of the original paper in Japanese $[72])$.

[74] Takahasi, H. and Mori, M., New method of error estimation in the numerical integration of analytic functions (in Japanese), Kokyuroku RIMS, Kyoto Univ., 91 (1970), 119-141.

[75] - Error estimation in the numerical integration of analytic functions, Report Comput. Centre, Univ. Tokyo, 3 (1970), 41-108.

[76] Error estimation in the numerical quadrature of analytic functions, Appl. Anal., 1 (1971), 201-229.

[77] L Quadrature formulas obtained by variable transformation (in Japanese), Kokyuroku RIMS, Kyoto Univ., 149 (1972), 93-110.

[78] — Quadrature formulas obtained by variable transformation, Numer. Math., 21 (1973), 206-219.

[79] , Quadrature formulas obtained by variable transformation (2) (in Japanese), Kokyuroku RIMS, Kyoto Univ., 172 (1973), 88-104.

[80] , Double exponential formulas for numerical integration, Publ. RIMS, Kyoto Univ., 9 (1974), 721-741.

[81] Tanaka, K., Sugihara, M. and Murota, K., Numerical indefinite integration by double exponential sinc method, Math. Comp., 74 (2004), 655-679.

[82] Toda, H. and Ono, H., Some remarks for efficient usage of the double exponential formulas (in Japanese), Kokyuroku RIMS, Kyoto Univ., 339 (1978), 74-109.

[83] Watanabe, T., On the double exponential formula for numerical integration (in Japanese), Kakuyugokenkyu, 63 (1990), 397-411.

[84] Yamamoto, Y., Double-exponential fast Gauss transform algorithms for pricing discrete lookback options, to appear in Publ. RIMS, Kyoto Univ., the present issue (2005). 